\title{
Investigating the role of emotion during the search process in free recall
}

\author{
Aisha P. Siddiqui • Nash Unsworth
}

Published online: 29 June 2011

(C) Psychonomic Society, Inc. 2011

\begin{abstract}
Typically, research has shown that emotional words are remembered better than neutral words; however, most studies have reported only the mean proportion of correctly recalled words. The present study looked at various dependent measures used by search models to determine whether emotion can influence the search process as well. The results from Experiment 2 showed that when emotionality was made salient, participants were able to utilize emotional associations, in addition to temporal associations, to cue retrieval of additional emotional words during subsequent sampling but relied mainly on temporal context when the emotional information was not made salient (Experiment 1). Additionally, both experiments showed that emotional words were more likely to be output earlier in the recall sequence, which would suggest that emotion also serves to boost relative strength during initial sampling. Overall, the results suggest that emotion contributes to enhanced memory dynamically by influencing the probability of sampling an item during the search process - specifically, by boosting relative strength and strengthening interitem associations.
\end{abstract}

Keywords Emotion $\cdot$ Search models $\cdot$ Free recall

The authors thank Dr. Gene Brewer and Greg Spillers for their helpful comments on earlier versions of the manuscript.

\footnotetext{
A. P. Siddiqui $(\bowtie) \cdot$ N. Unsworth

Department of Psychology, University of Georgia, Athens, GA 30602-3013, USA

e-mail: aps23@uga.edu

Present Address:

N. Unsworth

Department of Psychology, University of Oregon, Eugene, OR 97403, USA
}

Emotional items tend to be remembered better than nonemotional, or neutral, items. We experience this emotional enhancement of memory in everyday life-for instance, when one vividly recalls scenes from a scary movie to a friend or when one tries to remember details about one's first crush. Similarly, in controlled experimental settings, this memory advantage can be observed using various memory tasks (e.g., recognition and free recall tasks, etc.), as well as with different types of stimuli (e.g., pictures, Bradley et al., 1992; words, Maddock \& Frein, 2009, and Maltzman et al., 1966; auditory, Bradley \& Lang, 2000). While it is not clear exactly how this enhancement occurs, some researchers have offered possible explanations. One predominant theory is that arousing stimuli lead to a narrowed focus of attention to central details of the item at encoding, which helps to prioritize relevant material and facilitate better recall of the item (Easterbrook, 1959; Levine \& Edelstein, 2009; Yiend, 2010). This focused attention elicited by arousing stimuli leads to better memory for details intrinsic to, or belonging to, the item, as compared with details considered extrinsic to the item (Mather, 2007). Alternatively, some researchers have suggested that improved memory performance is more a consequence of emotional items' increased semantic relatedness, which facilitates the use of organizational strategies by the participant (Doerksen \& Shimamura, 2001; Talmi \& Moscovitch, 2004).

One commonality across these theories is how they report enhanced memory performance. Typically, in free recall tasks, better memory performance is expressed by reporting the mean proportion of correctly recalled emotional items, as compared with neutral items. While informative, this tradition does not allow further exploration into how emotionality is utilized by the participant in these tasks; specifically, it does not allow us to investigate how 
emotion can influence the search process and whether it can also function as an organizing principle. In order to explore the emotional-enhancement-of-memory effect in further detail, we need to analyze other dependent measures previously shown to reflect differences in the search process. The present study examined the influence of emotion on the contents of memory within the framework of search models to determine whether the enhanced emotional memory is due, in part, to changes in the search process.

\section{Search process in free recall}

Before delving into research on the emotional enhancement effect, it may be beneficial briefly to review the basic search model framework. In general, search models argue that information is retrieved from long-term memory through a competitive search process, in which information or features associated with sought-after items are intermixed with irrelevant information (Unsworth, 2009). One must use both the information that separates an item from others and the information that joins, or associates, two pieces of information. This point is relevant to the present article because it highlights two areas where the influence of emotion could be taking place.

An example of a long-standing search model is the search of associative memory model (Raaijmakers \& Shiffrin, 1980), which considers retrieval to be a probabilistic cue-dependent search of associative long-term memory network. According to these models, a general question asked by the participant (e.g., what were the words presented on the most recent list?) sets up the search context, while various retrieval cues are used to probe memory for additional information. Once a search set has been established, participants sample item representations from the search set (with replacement) on the basis of a relative strength rule (Raaijmakers \& Shiffrin, 1980; Rohrer, 1996; Shiffrin, 1970; Unsworth, 2009). In this case, the probability of sampling an item given the list context depends on the strength of the item-cue association (i.e., the amount of overlap between features associated with the item and features of the cue), and therefore, items with the greatest relative strength, as compared with other items on the lists, will have the greatest probability of sampling.

Once sampled, certain features of an item become activated, and if enough of these features are activated, the item will be recovered into consciousness. Then the participant can determine whether the item was on the list or not, or whether it was already generated, and if determined to be correct, the participant will recall the item. Interestingly, subsequent sampling of items can now utilize this just-recalled item as a retrieval cue to further delimit the search set to information associated with this item, and the process repeats itself. Thus, the probability of subsequent sampling now depends on associations between items on the list, and an item with a strong association with the just-recalled item has the greatest probability of being sampled (Raaijmakers \& Shiffrin, 1980). Eventually, when this search set turns up too many retrieval failures, the participants might probe memory with another global question or decide to stop retrieval attempts altogether.

\section{Emotional enhancement of memory}

Conceivably, using the framework of search models of free recall, one could hypothesize that emotion would have its greatest influence during this search process by manipulating the contents of the search set. According to the dimensional approach of emotion (Lang et al., 1990), emotion can be measured on two main dimensions: arousal and valence. Arousal is a measure of how arousing or calming an item is to the participant, and valence is a measure of how pleasant or unpleasant an item is to a participant. Differences in how each dimension contributes to the emotional enhancement effect have been a fundamental empirical question in most emotional memory experiments. In particular, studies manipulating both arousal and valence suggest that the two dimensions have varying neural correlates and rely on different mental processes (Kensinger \& Corkin, 2004). Specifically, these authors suggested that participants relied on more automatic processes for high-arousal items, which depend on amygdalar-hippocampal connections, whereas participants relied on more self-generated, controlled processes for nonarousing negative words, which depend on a prefrontal cortexhippocampal network.

Additionally, there is a great debate among researchers in this field as to how exactly the emotional enhancement of memory effect works. However, these basic claims can be simplified into two realms of thought: one suggesting preferential selection of emotional items over neutral items, due to greater attentional resources allocated to these items (Kensinger, 2009; Mather, 2007), in conjunction with other factors or alone (i.e., within-object binding, distinctiveness, etc.), and another suggesting more efficient organizational strategies utilized by the participants due to the enhanced relational nature of emotional words (Doerksen \& Shimamura, 2001; Talmi \& Moscovitch, 2004). Each of these explanations has a different prediction concerning its influence on the search process. According to these models, if emotion serves to focus attention on the individual item (Kensinger, 2009; Mather, 2007), we would expect to see a preference for these stronger emotional items to be initially sampled over neutral 
items, in accordance to the relative strength rule (i.e., stronger items on a list of varying relative strengths will be more likely to be initially sampled, as compared with weaker items; Raaijmakers \& Shiffrin, 1980).

Alternatively, if emotionality serves to strengthen itemto-item associations and elicit more efficient organizational strategies, we would expect an increase in the probability of subsequent sampling. The relationship between the justrecalled item and other items on the list would be more apparent and, therefore, would further delimit the search set. In this way, the stronger the relationship between two items, the more likely it is that they will be recalled together (Bousfield, 1953; Howard \& Kahana, 2002).

In order to compare the two explanations from a search model standpoint, one would need to look at dependent measures other than the mean proportion of words recalled. In particular, one could measure factors influenced by changes in relative strength and determine whether changes in emotionality have similar responses. For instance, previous research on relative strength differences has shown that variability in the strength of items on the list can account for differences in output order, as well as for differences in average recall latencies (Rohrer, 1996; Wixted et al., 1997). Specifically, stronger items were more likely to be output earlier in the recall period and in earlier recall positions, while weaker items were more likely to be output later in the recall period and in later positions when the list contained items of variable strengths (e.g., mixedlist presentation; Wixted et al., 1997). Specifically, in some cases, negatively arousing emotional pictures have been shown to be recalled at the beginning of the output period, as compared with related neutral or random neutral pictures (Talmi, Luk, et al., 2007); however, some of the same authors did not find an effect of output order in a different study (Talmi, Schimmack, et al., 2007), nor did this positive finding include both positive and negative images.

Mather, Kensinger, and others have suggested that arousal benefits memory by focusing attention on withinobject associations, while attention has no influence or, in some cases, impairs memory for between-object associations (Kensinger, 2009; Mather, 2007). In other words, arousal leads to better memory for details associated with an item, as compared with item-to-item associations, or between-item details, by binding certain elements of emotional stimulus together while not affecting or, sometimes, interfering with the processing of other bound representations. Mather suggested that arousal serves to bind features of an object together that are best perceived as belonging to the same object (such as color, spatial location, etc.), which leads to greater memory of those features and that object. Recently, Schmidt et al. (2011) showed that high arousal benefitted memory for contextual details associated with the item - in this case, spatial location and temporal order. Thus, it could be a strengthening of the emotional item itself, relative to other, nonemotional items on a list, that results in the enhanced memory.

Alternatively, it has been suggested that enhanced emotional memory is due, at least in part, to a participant's ability to use organizational strategies during encoding or retrieval (Doerksen \& Shimamura, 2001; Talmi \& Moscovitch, 2004). A participant's ability to organize items on a list in a particular and more efficient manner could facilitate greater associations between items and, thus, could mediate the enhanced emotional recall effect. To test this suggestion, one could look at measures of output grouping, which should indicate whether emotional items benefit from and/or recruit greater organizational strategies. For example, Howard and Kahana (2002) found that the probability of transitioning between semantically related words during recall is greater than the probability of transitioning between nonsemantically related words. Therefore, recalling an emotional item could cue the retrieval of additional emotional items, leading to greater probabilities of transitioning between emotionally related words. Specifically, when two words have very strong semantic associations, the probability that the two words will be recalled together is greater than that for two words that are not as strongly associated.

When word lists were matched for semantic similarity, Talmi and Moscovitch (2004) found similar recall percentages between categorized neutral words and emotional words (with percent recall statistically greater for both word lists than for random, neutral words). While careful to acknowledge the possibility of other factors, the authors suggested that semantic relatedness could account for enhanced memory by facilitating the implementation of organizational strategies on the participants' part. In fact, a more recent study by the same authors suggested that attention and other cognitive strategies, in addition to semantic relatedness, could contribute to the effect (Talmi, Schimmack, et al., 2007). Additionally, Doerksen and Shimamura (2001) suggested that organizational or elaborative processes based on individual differences in autobiographical experience, and not simply semantic interitem associations, could help enhance recall of emotional words.

\section{The present study}

Overall, the primary goal of the present study was to investigate how emotion leads to an enhancement of memory, using a theoretical model from the free recall literature - in particular, search models. By reporting a variety of analyses other than mean proportion of correctly recalled words, we should be able to determine whether emotion serves to boost relative strength of an item in each 
mixed-list presentation, leading to a greater probability of initially sampling the item over the others. Or, conversely, we could determine whether emotion also acts to strengthen interitem associations by facilitating the use of organizational strategies, leading to a greater probability of subsequent sampling. It is important to note that these two outcomes are not mutually exclusive and could simply indicate multiple sources of influence during the search process, depending on the information present.

If emotional items were to be output first during recall, with shorter recall latencies, we would predict that the emotional enhancement of memory effect is due to emotional items receiving a boost in relative strength and, thereby, changing/increasing the probability of initial sampling. On the other hand, but not mutually exclusive, if the probability of recalling two similarly valenced items together (i.e., significant clustering) were greater than the other transition possibilities, we would predict that emotional items are recalled more because they facilitate the use of organizational strategies by strengthening interitem associations and, thereby, change/increase the probability of subsequently sampling an emotional word.

\section{Experiment 1}

\section{Method}

\section{Participants}

Forty-five (23 of them female) undergraduates from the University of Georgia participated for course credit. All participants signed standard Institutional Review Board consent forms.

\section{Materials}

Seventy-two words were chosen from the Affective Norms for English Words (ANEW; Bradley \& Lang, 1999): 24 pleasant, 24 unpleasant, and 24 neutral. Pleasant words were randomly chosen from all words with valence values greater than 7.75 and arousal values greater than 6.00 $\left(M_{\mathrm{V}}=8.2 ; M_{\mathrm{A}}=6.8\right)$. Unpleasant words were randomly chosen from all words with a valence score less than 2.25 and an arousal score greater than $6.00\left(M_{\mathrm{V}}=2.0 ; M_{\mathrm{A}}=\right.$ 6.6). Neutral words were randomly chosen from all words with valence scores between 4.5 and 5.5 and with arousal scores less than $4.00\left(M_{\mathrm{V}}=5.1 ; M_{\mathrm{A}}=3.5\right)$. Pleasant, unpleasant, and neutral word types did not vary on KučeraFrancis word frequency, number of letters, or imagery values, (all $p \mathrm{~s}>.05$; Clark \& Paivio, 2004).

Three lists of 24 words were created with 8 words from each valence category per list (i.e., 8 pleasant, unpleasant, and neutral words per list). Pseudorandomized word lists were created to ensure that the first word from each list would be a pleasant, unpleasant, or neutral word, and subsequent words were randomly chosen from all word types. Once the randomized word lists were created, all participants received the same word order within a list as other participants; however, counterbalancing of first-list presentation occurred between subjects (see Appendix 1 for the list of included words).

\section{Procedure}

Participants were assigned to one of three counterbalanced conditions. They were told that they would be participating in a memory experiment and that their task was to recall as many words as possible.

Two practice lists, consisting of 10 letters instead of words, were presented to familiarize the participants with the experimental procedure. Each letter (or word, in the experiment proper) was presented individually on the screen for $2 \mathrm{~s}$ each. Prior to recall during practice and experimental trials, a 60 -s distractor task was presented, in which 30 three-digit numbers appeared on the screen for $2 \mathrm{~s}$ each and participants had to write the digits from largest to smallest on a provided sheet of paper. Immediately after the distractor task, three centrally placed question marks (e.g., ???) appeared on the screen for $2 \mathrm{~min}$, indicating to the participants that it was time to recall as many words as possible from the justpresented list. Participants pressed Enter after each response to clear the screen and were instructed to try to recall words throughout the entire recall period.

\section{Results}

No counterbalancing effects were found across pseudorandomized word lists, so all data were merged and analyzed together, $F(4,84)=0.97, p>.40$. There was a significant main effect of list order, overall, $F(2,88)=$ $10.36, p<.001$. More words were recalled in the first list than in the second or third list, $p s<.01$. However, no interaction between list order and item valence was found, $F(4,176)=0.66, p>.05$. Consistent with previous studies showing emotional enhancement of memory, there was a main effect of item valence, $F(2$, $88)=6.37, p<.01$. On average (see Fig. 1), the mean proportion of positive $(M=.43, S E=.02)$ and negative $(M=.40, S E=.02)$ words recalled were significantly greater than the mean proportion of neutral words recalled $(M=.35, S E=.02), t(44)=3.19, p<.01$, and $t(44)=2.50$, $p<.05$, respectively. There was no significant difference between the mean proportion of positive and negative words recalled, $t(44)=1.26, p>.05$. 


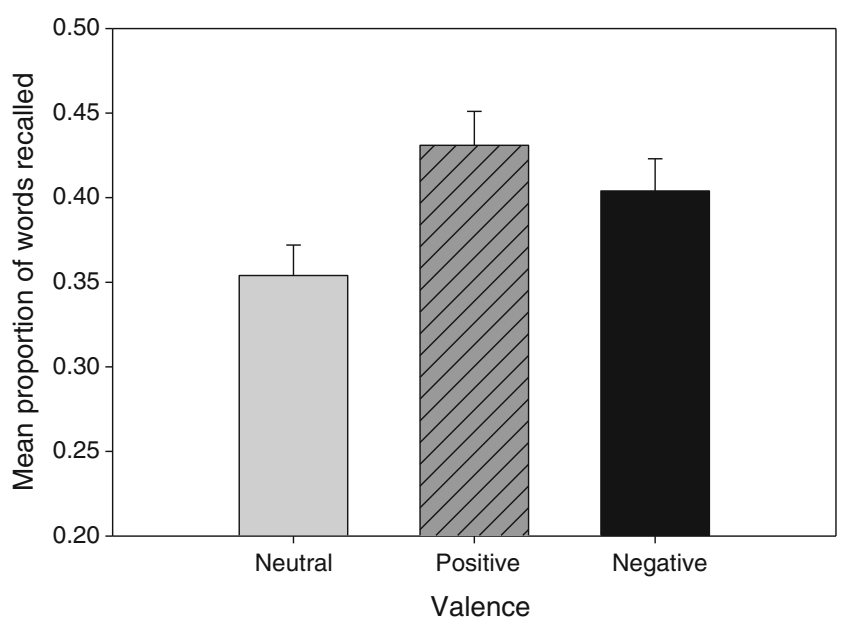

Fig. 1 Mean proportions of correctly recalled words by valence in Experiment 1. Error bars attached to each column represent standard errors

According to the first hypothesis, if emotion serves to boost relative strength, the probability of initial sampling should be increased, and we would expect emotional items to be recalled earlier in the recall sequence with shorter average recall latencies. We analyzed output order by rank ordering participants' responses into quintiles by list presentation. Each of the quintiles represented $20 \%$ of the output items per list computed by participant and then averaged across participants, which accounted for different numbers of recalled items across participants. The mean proportion of words recalled by valence at each quintile was calculated by taking the average number of words recalled per valence at each quintile and dividing it by the total of the average number of words recalled per valence across all quintiles. For instance, if the average number of neutral words recalled by a participant during the first quintile was 2 and the total number of neutral words recalled across all quintiles was 10 , the mean proportion of neutral words recalled at quintile 1 would be .2 , or $(2 / 10)$.

Similar to previous findings, the results indicate that stronger, emotional items tended to be recalled in earlier output quintiles, while weaker, neutral items tended to be recalled later in the sequence (see Wixted et al., 1997). A repeated measures ANOVA revealed a main effect of output quintile, $F(4,176)=25.0, p<.001$, and a significant interaction between item valence and output quintile, $F(8$, $352)=2.27, p<.05$. The proportion of negative item output was significantly greater than the proportion of neutral item output at the first, $t(44)=2.31, p<.05$, and second, $t(44)=$ $2.23, p<.05$, quintiles, while the proportion of neutral items was significantly greater than the proportion of negative items at the fourth quintile, $t(44)=2.31, p<.05$. While the other quintiles did not reach significance, a qualitative glance at Fig. 2 shows that the output curves for each valence

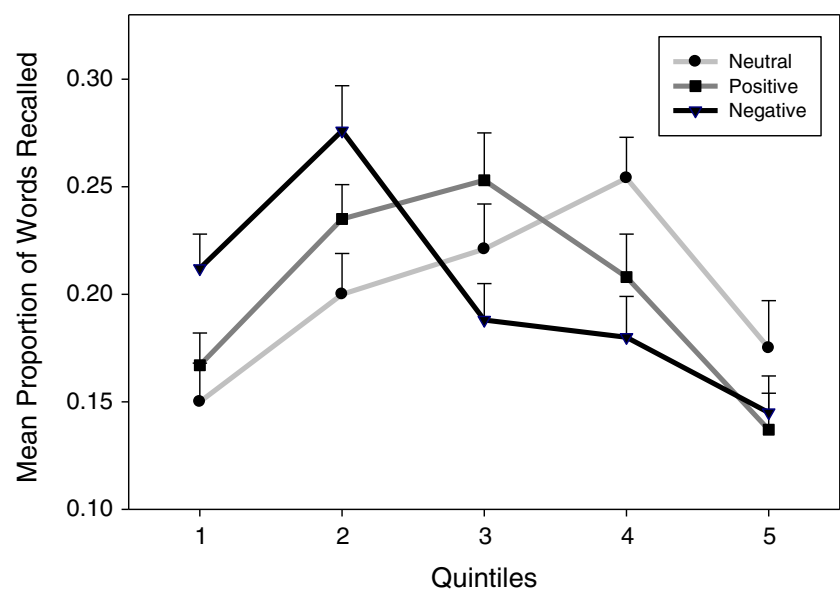

Fig. 2 Mean proportions of words recalled at each output quintile for Experiment 1, plotted separately by valence. Error bars at each data point represent standard errors

follow this pattern across quintiles. Negative items tend to be recalled in earlier output quintiles, peak at the second quintile, and then drop off. Positive items tend to be recalled following that, with a peak around the third quintile. Finally, neutral items tend to be recalled in the later output quintiles, with a peak around the fourth quintile.

Whereas output quintiles represent a rough estimate of when items are output, average recall latencies give a more quantitative measure of output order. Average recall latency was measured by calculating the difference in time from the onset of the recall period to average recall of each item type. For instance, if a participant recalled three negative items $(5,10$, and $15 \mathrm{~s}$ after the recall period began), the average recall latency for negative words would be $10 \mathrm{~s}$ for that participant. On the other hand, if another participant recalled three negative words at 10,15 , and $20 \mathrm{~s}$ after the recall period began, their average recall latency would be $15 \mathrm{~s}$, and the overall average recall latency for negative items would be $12.5 \mathrm{~s}$.

Overall, a significant main effect of item valence was found, $F(2,88)=4.12, p<.05$. Average recall latencies were shorter for negative items $(M=21.5 \mathrm{~s}, S E=1.2)$ and positive items $(M=23.8 \mathrm{~s}, S E=1.3)$ than for neutral items $(M=25.8 \mathrm{~s}, S E=1.3)$. Consistent with the output quintiles, these results indicate that negative items were output first, followed by positive items and, finally, neutral items (see Fig. 3). Individual $t$-tests confirmed that negative items had significantly shorter average recall latencies than did neutral items, $t(44)=3.03, p<.01$, but were not significantly different from positive items, $t(44)=1.57, p>.1$.

According to the second hypothesis, if emotional enhancement is due to emotion's increasing the probability of subsequent sampling by facilitating organizing strategies, we would expecte that emotional items would be recalled together more often. Transition probabilities indicate the 


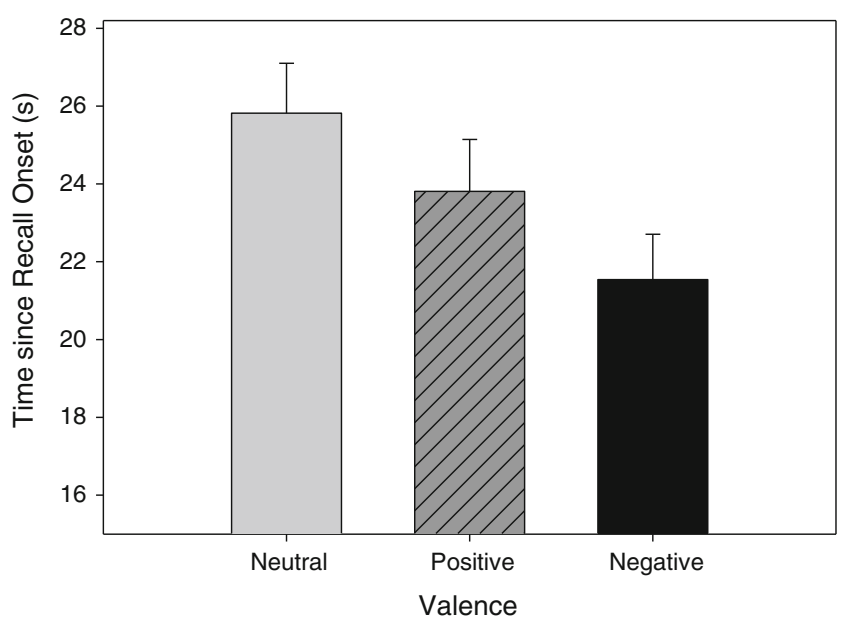

Fig. 3 Average recall latencies by valence in Experiment 1. Error bars attached to each column represent standard errors

probability of recalling a word of a certain item type, given recall of the same valence type or some other type (see Appendix 2 for calculating transition probabilities). Additionally, we report the adjusted ratio of clustering (ARC) for each valence type, which provides additional empirical evidence of above-chance category repetitions without the influence of unrelated factors (Roenker et al., 1971).

Table 1 shows the transition probabilities for each item type. On average, when participants recalled a negative or neutral item, they were equally likely to subsequently recall an item of any valence, as indicated by probabilities near chance $(0.333$, or $1 / 3)$. Chi-square values indicated no significant differences across neutral transitions, $\chi^{2}(2)=1.52, p>.05$, as well as no significant differences across negative transitions, $\chi^{2}(2)=2.89, p>.05$. However, positive transitions were significantly different from each other, $\chi^{2}(2)=10.88, p<.01$. A look at Table 1 indicates that positive to positive transitions were slightly above chance levels and positive to neutral transition were slightly below chance levels.

Correspondingly, ARC scores show results similar to the transition probabilities. There was a main effect of emotion type, $F(2,88)=5.162, p<.01$. Paired samples $t$-tests showed that positive clusters $(M=0.15, S E=0.01)$ were significantly greater than negative clusters $(M=0.13, S E=$ $0.01), t(44)=2.23, p<.05$, and neutral clusters $(M=0.12$, $S E=0.01), t(44)=2.94, p<.01$. No significant differences

Table 1 Transition probabilities for Experiment 1

\begin{tabular}{llll}
\hline Item & Neutral & Positive & Negative \\
\hline Neutral-to- & .347 & .300 & .353 \\
Positive-to- $*$ & .261 & .395 & .344 \\
Negative-to- & .310 & .375 & .315 \\
\hline
\end{tabular}

${ }^{*} p<.05$ were found between negative and neutral clusters, $t(44)=0.86, p>.3$.

Overall, the transition probabilities suggest that participants were not utilizing emotional categorical membership as a tool to facilitate recall of additional items, or if they were (i.e., positive transitions), the strength of the semantic associations were not strong enough to have transitions exceedingly well over chance. This indicates that perhaps some other type of association drives subsequent sampling; but what?

It seems possible that participants could be using temporal associations to increase subsequent sampling. Fig. 4 shows the lag recency functions for forward and backward transitions. These functions represent the probability of forward and backward transitions made between correctly recalled items on the basis of presentation lag. For instance, previous research on conditional response probabilities (CRPs) indicates that it is more likely to recall two words together that were immediately presented together (lag of 1) than when they appeared farther apart on the presentation list (lag greater than 1) and more likely to recall words in a forward direction rather than backward (Howard \& Kahana, 1999, 2002; Kahana, 1996; Unsworth, 2008). In other words, if the 9th item on the list was recalled, participants were more likely to subsequently recall the 10th item on the list, rather than the 8th item (i.e., recall proceeds in a forward direction) or the 24th (i.e., recall probability of closely presented items is greater), regardless of valence.

Lag-CRPs were calculated using the same method as prior researchers (Howard \& Kahana, 1999; Kahana, 1996; Unsworth, 2008). Consistent with prior research, we found significant main effects of direction (forward $>$ backward), $F(1,44)=7.24, p<.01$, and lag $(1>2-5), F(4,176)=$ $18.69, p<.001$, as well as a significant interaction of lag and direction, $F(4,176)=12.07, p<.001$. These results

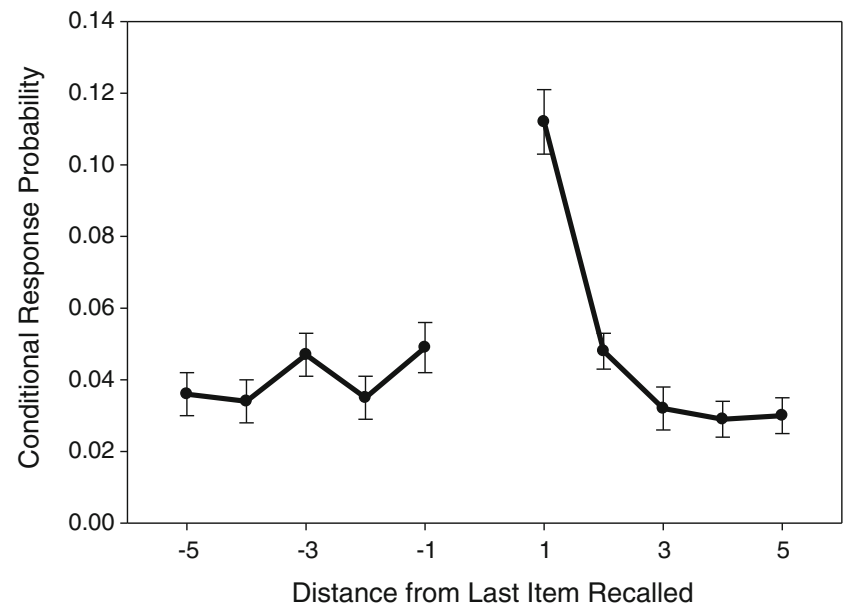

Fig. 4 Lag-CRP for Experiment 1. Error bars attached to data points represent standard errors 
indicate that the next word participants stated after recalling a word was most likely a word originally appearing immediately after that one in the presentation list; recall proceeded in a forward direction with a lag of 1 .

\section{Discussion}

Overall, the results from the first experiment suggest that emotion enhances memory recall by influencing initial sampling of items during the search process but appears not to influence subsequent sampling significantly. Positive and negative words were more likely to be recalled earlier in the output sequence and were more likely to have shorter average recall latencies, which would suggest that emotional items received a boost in relative strength, as compared with neutral items. On the other hand, transition probabilities for negative and neutral transitions indicated that participants were equally likely to recall a word with the same valence as the previously recalled word as they were an item with a different valence. However, participants were able to use temporal context as a cue for subsequent sampling and recalled items that were presented in nearby positions. Thus, subsequent sampling depended more on temporal context than on emotional context. Specifically, participants were able to use the emotionality of an item to initiate the recall sequence but were more likely to use temporal associations, instead of semantic associations, to cue recall of additional words.

However, in the case of positively related words, participants were able to use the emotional context of the word to cue recall to some extent, as indicated by the significant positive-to-positive transition probabilities. Thus, the following questions arise. Were positive words somehow organized differently from neutral and negative words, such that a positive word could be used more efficiently as a retrieval cue, or was this just a Type I error? Or perhaps, was the emotionality of positive words somehow more salient to the participants than negative words, which enabled them to primarily limit their search set to positive words? Or alternatively, was the lack of significant transition probabilities for negative items a Type II error?

Brosch et al. (2010) suggested that categorization of emotional stimuli is a learned, adaptive construct that is flexible and changing. We group or classify stimuli utilizing top-down processes subject to contextual demands, such as experimenter demands or individual differences. Likewise, Sison and Mather (2007) suggested that categorical membership is a flexible and context-dependent structure that relies on the most salient organizational schema and is not a rigid system that gives priority to emotional categories. Using a part-set cuing paradigm, they presented pictures to participants that could be categorized along an emotional categorical structure (amusing vs. fearful), as well as being categorized as people or animals. They showed that competition among same-category items exhibited the typical interference effect when the category of interest was highlighted initially in the instructions. Interestingly, they found that the interference effect for the alternative category went away, even though the words were the same. In other words, when the initial instructions mentioned people versus animal categories, recall of noncued members was impaired, and when the instructions mentioned amusing versus fear, recall of noncued emotionmatch pictures was impaired. As a result, they concluded that emotional categories are not special but can act just like other categories that compete for resources during retrieval.

Thus, according to Brosch et al.'s (2010) review and Sison and Mather's (2007) study, perhaps, participants in our study were able to utilize emotion as an organizing principle, but our instructions did not allow the emotionality of all words to be salient enough to group upon. Specifically, by not specifying beforehand that participants would be seeing emotional words, we could have reduced their ability as a whole to effectively use emotion as a retrieval cue and readily utilize the emotional information to cluster. Or, alternatively, the words chosen for the study may not elicit the same level of pleasantness or unpleasantness for every participant, which would influence how they organize or categorize emotional words. For instance, while we controlled for the mean valence and arousal of each item, perhaps, on an individualistic level, the words were not as emotional to the participant. Perhaps, the word pollute is not considered very unpleasant to some participants, so it would be less likely to be "bound" with other negative words and, thereby, less likely to be an effective retrieval cue.

\section{Experiment 2}

In Experiment 2, we had participants give pleasantness ratings for each item at encoding, in order to make the emotionality salient to each participant. If emotion is able to facilitate organizational strategies for positive and negative words but, somehow, our experimental design weakened our ability to detect it, highlighting the emotionality of an item should increase transition probabilities between similarly valenced items. However, if the results from the first experiment were simply a Type I error and organizational strategies do not contribute to emotional enhancement of memory, we would expect the transition probabilities for all transition types to remain at chance. Additionally, by recording participants' individual ratings of perceived pleasantness, we can extract those items that were perceived as moderately or minimally emotional and investigate how perceiving an emotion more intensely influences memory processes. 


\section{Method}

\section{Participants}

Thirty-nine (21 of them female) undergraduates from the research pool at the University of Georgia participated in this experiment for partial fulfillment of course credit. For simplicity, we did not split participants into conditions, since there were no effects of list counterbalancing effects in the previous experiment.

\section{Materials and procedure}

All stimuli and instructions were the same as in the first experiment, with a few exceptions. Participants in the second experiment were explicitly told that some items would have an emotional meaning, while some items would not. During the study phase, they were instructed to make pleasantness ratings for each of words while the word was presented on the screen. They rated items on a 5-point scale ( 1 =highly unpleasant and $5=$ highly pleasant $)$ and then pressed Enter after making their response. Their rating appeared in a smaller font on the upper left-hand part of the screen away from the presentation of the word. To compensate for increased task complexity, the word was presented for $3 \mathrm{~s}$, instead of $2 \mathrm{~s}$. Responding did not alter the presentation timing of each word, and therefore, it was possible not to get ratings for a word if participants exceeded the allotted time without making a response (i.e., no rating would be recorded).

\section{Results}

Consistent with previous studies, as well as the first experiment, emotional words were recalled in greater proportions than were neutral words. As is shown in Fig. 5, a repeated measures ANOVA revealed a main effect of emotion, $F(2,76)=12.4, p<.001$. The mean proportion of positive $(M=.45, S E=.02)$ and negative $(M=.41$, $S E=.02)$ words recalled were significantly greater than the mean proportion of neutral words recalled $(M=.34, S E=$ $.02), t(38)=4.52, p<.001$, and $t(38)=3.89, p<.001$, respectively. There was no significant difference between the mean proportion of positive and negative words recalled, $t(38)=1.79, p>.05$.

Because we asked participants to give a pleasantness rating for each item, we could now look at how an individual's perceived pleasantness of an item affected recall. On average, $7.8(S E=0.09)$ neutral, $7.5(S E=0.05)$ positive, and 7.1 $(S E=0.17)$ negative words per list were given ratings. Analysis of the ratings indicated an interaction effect between rating value and emotion type, $F(8,304)=$ $196.95, p<.001$. Participants were much more likely to give neutral words a rating of $3(M=0.63, S E=0.03)$, as

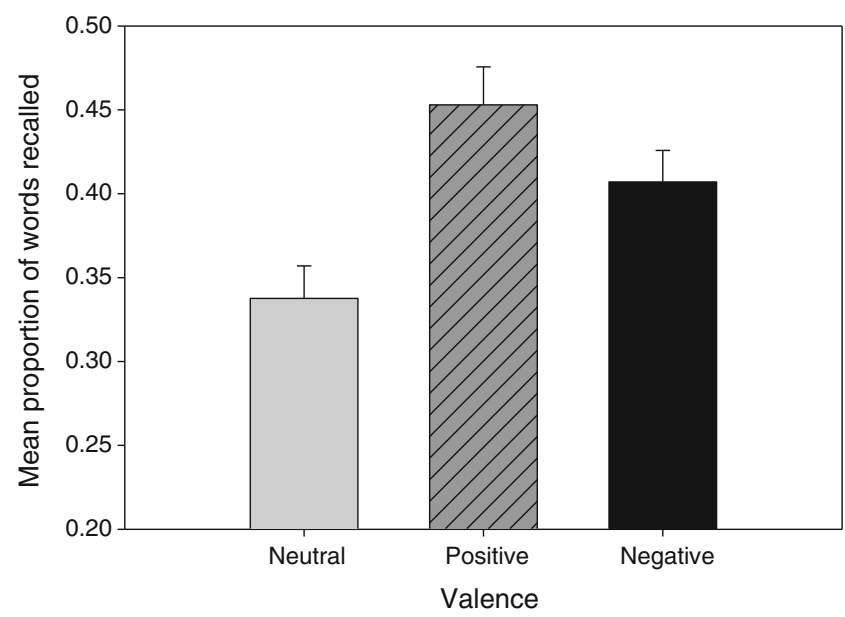

Fig. 5 Mean proportion of correctly recalled words by valence in Experiment 2. Error bars attached to each column represent standard errors

compared wih any other value $(M \mathrm{~s}<0.15)$. Positive words were much more likely to be given the extreme corresponding valence rating $(M=0.69, S E=0.04)$, as compared with a moderate rating $(M=0.22, S E=0.03)$ or any other value, $(M \mathbf{s}<0.07)$. Negative words were much more likely to be given the extreme corresponding valence rating $(M=0.77, S E=0.04)$, as compared with a moderate rating $(M=0.14, S E=0.03)$ or any other value, $(M \mathbf{s}<0.06)$. Given that words were selected to be high in both arousal and valence, this preference for the extreme corresponding valence rating is intuitive. Furthermore, these results indicate that participants were able to perform the rating task properly and accurately.

Additionally, a significant main effect of emotion rating was observed concerning the probability of recalling an item given a particular rating, $F(4,136)=7.8, p<.001$. When an item was rated as highly unpleasant or highly pleasant (e.g., rating of 1 or 5 , respectively), they were more likely to recall the item during retrieval $(M=0.40, S E=0.02$, and $M=0.51$, $S E=0.03$, respectively), as opposed to when they rated the item as neutral (e.g., rating of $3 ; M=0.31, S E=0.02$ ), moderately unpleasant (e.g., rating of $2 ; M=0.33, S E=$ 0.05 ), or moderately pleasant (e.g., rating of $4 ; M=0.35$, $S E=0.03)$. These results indicate that those items rated as moderately unpleasant or moderately pleasant could have weakened the transition probabilities from the previous experiment. Because they were perceived as being less pleasant or unpleasant than the other items, perhaps they were not as strongly associated together as the other highly rated items and, thereby, decreased the possibility of being utilized as a retrieval cue. Therefore, for the remainder of the analyses, we will report data only on those items rated as highly pleasant, highly unpleasant, or neutral.

As in the first experiment, we analyzed output positions and recall latencies. Output quintiles were analyzed as a function of 
only three valence types and their respective ratings: neutral (3), highly unpleasant (1), and highly pleasant (5). According to Fig. 6 and consistent with the first experiment, there was a significant main effect of output quintile, $F(4,152)=6.88$, $p<.001$, and a marginally significant interaction of output position and emotion rating, $F(8,304)=1.72, p<.09$. While the interaction was only marginally significant, the pattern is clear: Emotional items (those rated as highly pleasant or unpleasant) are more likely to be output in earlier quintiles, as compared with neutral items; then, around the third quintile, they switch, and neutral items are more likely to be recalled. To further explore this relationship, we collapsed the pleasant and unpleasant output functions into one overarching emotional output curve. In other words, we looked at the output quintile curves as a function of emotional content (highly emotional: highly pleasant and highly unpleasant vs. neutral), instead of as a split function of emotional valence. This main effect, as well, is close to conventional levels of significance, $F(4,152)=2.36, p<.06$. As can be seen in Fig. 7 , it is clear that emotional words come out earlier in the recall sequence, whereas neutral words are more likely to come out later in the sequence.

Likewise, the average recall latencies (see Fig. 8), which represent a more sensitive measure of output order, correspond with the output quintiles. There was a significant main effect of emotion type, $F(2,76)=4.97, p<.01$. Overall, average recall latencies were shorter for highly unpleasant items $(M=28.0 \mathrm{~s}, S E=1.8)$ and highly pleasant items $(M=26.1 \mathrm{~s}, S E=1.5)$ than for neutral items $(M=$ $33.9 \mathrm{~s}, S E=2.6$ ). Individual $t$-tests confirmed that there were no significant differences between the average recall latencies of negative and positive items, $t(38)=1.10$, $p>.05$, but both were significantly shorter than the average

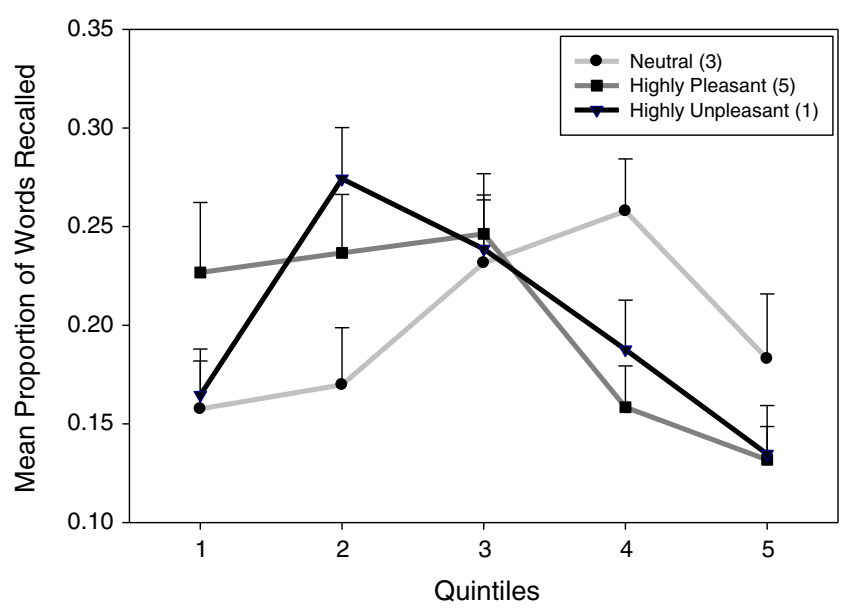

Fig. 6 Mean proportion of words recalled at each output quintile for Experiment 2, plotted separately by valence. Highly pleasant and highly unpleasant functions represent items given an extreme valence rating of 5 or 1, respectively. Error bars at each data point represent standard errors

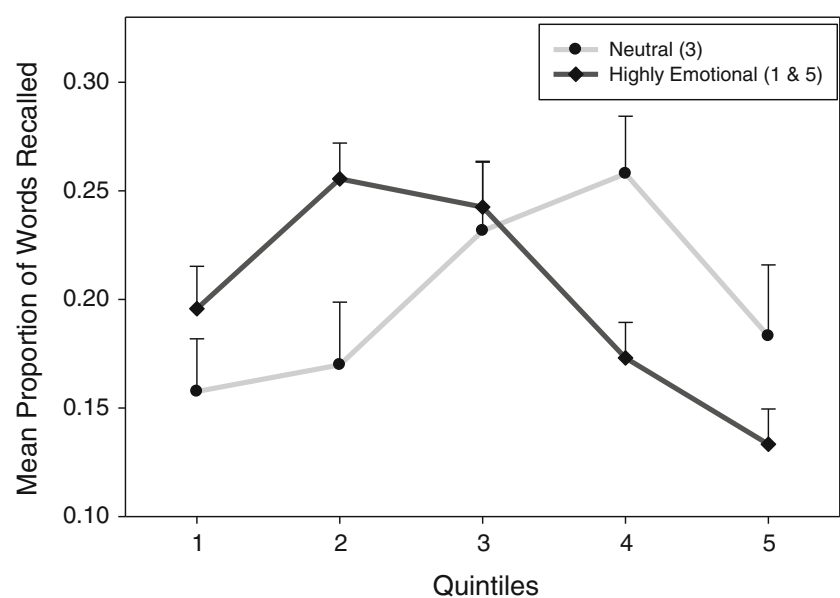

Fig. 7 Mean proportions of words recalled at each output quintile for Experiment 2, plotted separately by valence. Highly emotional function represents items given an extreme valence rating of 1 or 5 . Error bars at each data point represent standard errors

recall latencies of neutral items, $t(38)=2.02, p<.05$, and $t(38)=2.79, p<.01$, respectively. These results indicate that highly pleasant and unpleasant words were more likely to be output first, followed by neutral items.

Overall, the output quintiles and recall latencies suggest that, like before, the emotional enhancement effect is due, in part, to emotion's influencing the search process and receiving a boost in relative strength. However, the pivotal question concerning Experiment 2 was whether output grouping would be influenced when the emotional content-specifically, the pleasantness level-was made salient. We were especially interested in whether making emotionality salient would increase transition probabilities between items with a similar valence by enabling participants to utilize organizational strategies and whether perceived pleasantness would influence this effect on an individual level.

Table 2 shows the transition probabilities for each item type. On average, when participants recalled a neutral item, they were equally likely to subsequently recall an item of any valence, as indicated by probabilities near chance (.333, or $1 / 3)$. Particularly, the results show that there were no significant differences across neutral transitions, $\chi^{2}(2)=$ $2.45, p>.05$, but there were significant differences across negative transitions, $\chi^{2}(2)=7.30, p<.05$, and positive transitions, $\chi^{2}(2)=31.53, p<.001$. Table 2 indicates that negative-to-negative transitions were above chance levels and that negative-to-neutral transitions were below chance levels, while positive-to-positive transitions were above chance levels, as well as greater than in the first experiment, and positive-to-neutral transitions were below chance levels. This indicates that the lack of significance in the first experiment for negative transitions was likely due to our experimental instructions, which did not emphasize the emotional categorical structure that would have facilitated 
Fig. 8 Average recall latencies by rating in Experiment 2. Error bars attached to each column represent standard errors

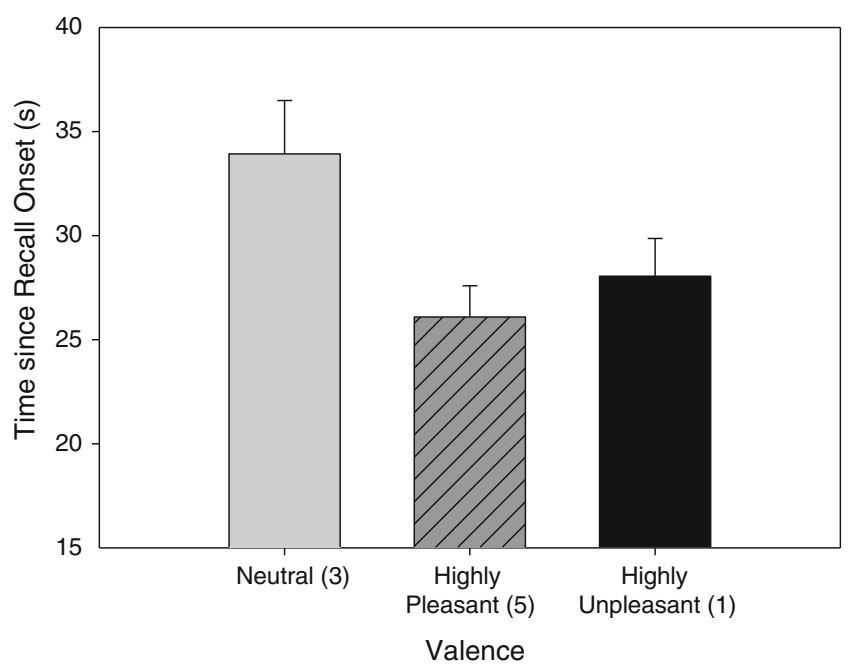

$0.01), t(38)=2.07, p<.05$, and neutral clusters $(M=0.08$, $S E=0.01), t(38)=4.28, p<.001$. Negative clusters were significantly greater than neutral clusters, $t(38)=2.12, p<.05$.

In general, these results suggest that the emotionality of an item was not readily available to use actively and efficiently as a retrieval cue in Experiment 1; however, enhancing the saliency of the emotionality enabled participants to utilize the semantic and categorical associations between items to facilitate retrieval. Nonetheless, if participants are now able to utilize the semantic associations between items, how does this influence their previous reliance on temporal associations, as suggested in Experiment 1? As can be seen in Fig. 9, the overall pattern for the lag-CRPs is the same as before: Participants are most likely to make forward transitions with a lag of $1, F(4,152)=3.87, p<.01$. However, while this pattern is the same, the probability of making a +1 forward transition is greatly reduced in Experiment 2, as compared with Experiment $1(M=0.06$, $S E=0.01$ vs. $M=0.11, S E=0.01$, respectively), $F(4,328)=3.91, p<.005$. This result indicates that participants were still able to utilize temporal associations in order to delimit the search set for subsequent sampling, but their reliance mainly on temporal associations was diminished to compensate for the now stronger semantic associations between items.

Table 3 Transition probabilities by rating for Experiment 2

Table 2 Transition probabilities for Experiment 2

\begin{tabular}{llll}
\hline & Neutral & Positive & Negative \\
\hline Neutral-to- & .360 & .288 & .352 \\
Positive-to- $*$ & .236 & .468 & .296 \\
Negative-to- $*$ & .266 & .342 & .392 \\
\hline
\end{tabular}

${ }^{*} p<.05$

\begin{tabular}{llll}
\hline & Neutral (3) & $\begin{array}{l}\text { Highly } \\
\text { Pleasant (5) }\end{array}$ & $\begin{array}{l}\text { Highly } \\
\text { Unpleasant (1) }\end{array}$ \\
\hline Neutral (3)-to- & .356 & .356 & .288 \\
Highly pleasant (5)-to- * & .179 & .489 & .332 \\
Highly unpleasant (1)-to- * & .219 & .365 & .416 \\
\hline
\end{tabular}

$* p<.05$ 


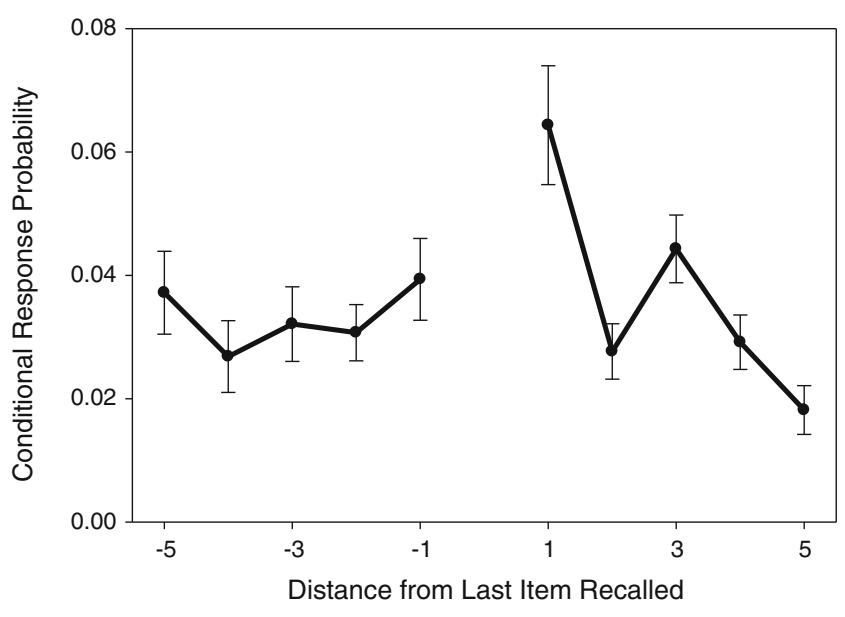

Fig. 9 Lag-CRP for Experiment 2. Error bars attached to data point represent standard errors

\section{Discussion}

The results from the second experiment suggest that participants, when made aware of the pleasantness of an item, could use that factor to cue recall of additional emotional words. Specifically, the results showed that the significant transition probability in Experiment 1 for positive-to-positive transitions was less likely to be a Type I error. They also showed that we can most likely rule out the possibility of positive items having privileged access to some grander organizational schema, since negative-to-negative transitions became significant as well. By highlighting the pleasantness, we showed that the nonsignificant transition probabilities from the previous experiment were most likely due to individual differences in how participants chose to organize items, consistent with the notion that emotional categories are flexible and dynamic constructs (Brosch et al., 2010; Sison \& Mather, 2007). Furthermore, by recording pleasantness ratings, it allowed us to measure how perceived differences in emotionality could influence recall; namely, we found that participants could now utilize emotionality as a cue for organizing highly pleasant and highly unpleasant words together. Not only did the probability of transitioning to a similarly valenced word increase for positive and negative words, but when we specifically looked at those words perceived as most pleasant, or unpleasant, we found that those probabilities increased more so for emotional words than for neutral words.

\section{General discussion}

Overall, from the two experiments in this study, we found that emotion contributes to enhanced memory by influencing the probability of sampling an item during the search processspecifically, by boosting relative strength and strengthening interitem associations. Experiment 1 showed that positive and negative words were output first in the recall sequence, indicating that emotional items were stronger relative to their neutral counterparts. The greater the relative strength, the more likely it is that the item will be sampled initially. On the other hand, subsequent sampling appeared not to be affected in the first experiment, as indicated by transition probabilities near chance. Thus, Experiment 1 suggested that emotion leads to enhanced memory recall by boosting relative strength of emotional words, relative to neutral words on the list, rather than by strengthening interitem associations between emotional words.

However, it was possible that not explicitly telling participants that they were going to see emotional words, we reduced their ability to effectively utilize emotion as a retrieval cue. Therefore, in Experiment 2, we had participants make pleasantness ratings on each item, in order to make the emotionality salient to each participant. We found that when emotional items were perceived as highly pleasant or highly unpleasant, they were more likely to be output first, consistent with Experiment 1, but now they were able to utilize the semantic associations existing between emotional words to delimit the search set and subsequently sampling from mostly emotional words. Participants began recall with a highly emotional word and then used that word to search for other highly emotional words. When that search cycle ended, participants started recalling weaker, neutral words, which, by that time, occur much later in the recall sequence. As indicated by the equivalent transition probabilities for neutral transitions, participants then recalled any word type subsequently, regardless of valence. Thus, it appears that the emotional enhancement of memory effect is due, in part, to emotion's influence on the probability of sampling during search process.

Our results support previous theories of emotional enhancement of memory - namely, those theories that suggest that emotional items are preferentially selected over other items and those theories that suggest that emotional items have stronger item-to-item associations (Talmi, Luk, et al., 2007). First, we were able to show successfully in both experiments the typical emotional enhancement effect, in which emotional words (both positive and negative) were recalled in greater mean proportions than neutral items. Second, when using both highly arousing negative and positive words, as well as neutral words, we were able to replicate previous findings showing joint contributions of distinctiveness and organization in memory for negatively arousing scenes (Talmi, Luk, et al., 2007; see also Hunt \& McDaniel, 1993).

Theories claiming that emotional items receive greater attentional resources (Easterbrook. 1959; Mather, 2007; S. R. Schmidt \& Saari, 2007), whether solely or in conjunction with other factors, propose that emotional items are somehow preferentially selected among other items on the list. If this 
were true, the additional attentional resources emotional items recruit at encoding could preferentially increase rehearsal times of emotional items, relative to neutral items, thereby creating stronger, emotional items and weaker, neutral items. In turn, we not only would see a greater proportion of emotional words recalled, but also would see emotional words recalled earlier in the recall sequence. In both Experiments 1 and 2, we showed that emotional items were more likely to be output earlier in the recall period, suggesting that the probability of initial sampling was influenced by emotional items receiving a boost of relative strength.

One interesting implication of the finding that emotion boosts relative strength is that it could possibly account for why memory is enhanced more when emotional and neutral words are presented in a mixed-list design, as opposed to a between-list design (S. R. Schmidt \& Saari, 2007, Experiment 3). According to Wixted et al. (1997), when all items have the same relative strength, the probability of sampling a specific item is equal to the probability of sampling any other item on the list. Therefore, when you give participants a list of all emotional words and a list of all neutral words, the probability of sampling an item on either list is equivalent. Moreover, as is shown in the present study, when the word list has both emotional and neutral words together, the variability in strengths leads to a greater probability of sampling stronger, emotional words, as compared with weaker, neutral words.

On the other hand, organizational theories propose that emotional items elicit more efficient organizational strategies and strengthen associations between items (Doerksen \& Shimamura, 2001; Talmi \& Moscovitch, 2004; Talmi, Schimmack, et al., 2007). If this were true, we should see more grouping of emotional words during recall, similar to how items with stronger semantic associations are more likely to be sampled and recalled together, as compared with nonsemantically related items (Bousfield, 1953; Howard \& Kahana, 2002). Since emotional categorization is a flexible and dynamic construct (Brosch et al., 2010; Sison \& Mather, 2007), when we made the emotionality salient in Experiment 2, participants could utilize the inherent categorical structure to sample from all words with emotional associations. However, when this association was not explicit, as in Experiment 1, participants were more likely to utilize temporal associations to sample from subsequently. In this case, we found in the second experiment that similarly valenced emotional items were more likely to be recalled together, as opposed to any other valence, and interestingly, those items perceived as being highly pleasant or unpleasant were even more likely to be recalled together. This finding suggests that individual differences in how a participant perceives an item could also affect how the item will potentially be organized.

If emotionality is capable of acting like a category (e.g., Bower, 1981; Niedenthal et al., 1999), attention to features of the category (i.e., emotional features) would increase clustering. This result is corroborated by work by Polyn, Kahana, and colleagues in their context maintenance and retrieval (CMR) model (Polyn et al., 2009a, 2009b; Sederberg et al., 2010). In the CMR model, categorical items that are attended to at encoding increase clustering at recall. These effects match those in the present study. Future research could extend the emotion as a category hypothesis by examining clustering and switching using the CMR model.

Overall, we propose that the effects of emotion on memory reflect the separation and strengthening of emotional items over neutral items in a word list, as well as the strengthening of associations between emotional items perceived as having the same level of emotionality. Together, the strengthening of emotional items over neutral items and the strengthening of associations between emotional items lead to a greater probability of recalling emotional words, as compared with neutral words.

\section{Appendix 1}

\begin{tabular}{llllll}
\hline LIST 1 & & LIST 2 & & LIST 3 & \\
(FirstWord Neutral) & (First Word Pleasant) & (First Word Unpleasant) \\
\hline VIOLIN & $\mathrm{n}$ & HAPPY & $\mathrm{p}$ & RABIES & $\mathrm{u}$ \\
VICTIM & $\mathrm{u}$ & TERRIFIED & $\mathrm{u}$ & PROMOTION & $\mathrm{p}$ \\
JOY & $\mathrm{p}$ & WAR & $\mathrm{u}$ & DISLOYAL & $\mathrm{u}$ \\
HAIRPIN & $\mathrm{n}$ & BUS & $\mathrm{n}$ & LOCKER & $\mathrm{n}$ \\
JOKE & $\mathrm{p}$ & LAWN & $\mathrm{n}$ & PAIN & $\mathrm{u}$ \\
BANDAGE & $\mathrm{n}$ & CONFIDENT & $\mathrm{p}$ & ROMANTIC & $\mathrm{p}$ \\
HATE & $\mathrm{u}$ & GIFT & $\mathrm{p}$ & PAPER & $\mathrm{n}$ \\
MOTHER & $\mathrm{p}$ & SLAVE & $\mathrm{u}$ & SLAUGHTER & $\mathrm{u}$ \\
WIN & $\mathrm{p}$ & AFFECTION & $\mathrm{p}$ & BOARD & $\mathrm{n}$ \\
TABLE & $\mathrm{n}$ & KILLER & $\mathrm{u}$ & PARTY & $\mathrm{p}$ \\
UMBRELLA & $\mathrm{n}$ & DROWN & $\mathrm{u}$ & SUCCESS & $\mathrm{p}$ \\
CRUCIFY & $\mathrm{u}$ & KISS & $\mathrm{p}$ & ELBOW & $\mathrm{n}$ \\
QUART & $\mathrm{n}$ & REVERENT & $\mathrm{n}$ & IRON & $\mathrm{n}$ \\
FEARFUL & $\mathrm{u}$ & SQUARE & $\mathrm{n}$ & CASH & $\mathrm{p}$ \\
STRESS & $\mathrm{u}$ & TREASURE & $\mathrm{p}$ & ACCIDENT & $\mathrm{u}$ \\
SEXY & $\mathrm{p}$ & LAMP & $\mathrm{n}$ & CHEER & $\mathrm{p}$ \\
CANCER & $\mathrm{u}$ & STREET & $\mathrm{n}$ & RESERVED & $\mathrm{n}$ \\
LAUGHTER & $\mathrm{p}$ & ABUSE & $\mathrm{u}$ & GRADUATE & $\mathrm{p}$ \\
PRETTY & $\mathrm{p}$ & LOVE & $\mathrm{p}$ & ECSTASY & $\mathrm{p}$ \\
VICTORY & $\mathrm{p}$ & THRILL & $\mathrm{p}$ & DEVIL & $\mathrm{u}$ \\
METAL & $\mathrm{n}$ & RAIN & $\mathrm{n}$ & ULCER & $\mathrm{u}$ \\
BOWL & $\mathrm{n}$ & POLLUTE & $\mathrm{u}$ & BENCH & $\mathrm{n}$ \\
DISASTER & $\mathrm{u}$ & POISON & $\mathrm{u}$ & SPHERE & $\mathrm{n}$ \\
TOXIC & $\mathrm{u}$ & KETTLE & $\mathrm{n}$ & ASSAULT & $\mathrm{u}$ \\
\hline & & & & & \\
\hline
\end{tabular}




\section{Appendix 2}

\section{Calculation of transition probabilities}

Transition probabilities were computed by calculating the probability of recalling one item type followed by the same item type, as well as for the other valences (i.e., the probability of recalling a positive item followed by a positive item, or followed by a negative or neutral item). These probabilities were computed separately for each valence. For instance, positive transitions (e.g., positive word followed by a positive, negative, or neutral word) were computed separately from negative transitions (e.g., negative word followed by a negative, positive, or neutral word) or neutral transitions (e.g., neutral word followed by a neutral, positive, or negative word). Comparisons across positive, negative, or neutral transitions (i.e., across rows in Tables 1-3) were not possible, since the ratio is dividing by different total transition values.

The total number of each type of transition (i.e., total number of positive-to-positive transitions, total number of positive-to-negative transitions, etc.) was calculated for each participant. This value was then divided by the total number of transitions between each item type (i.e., total number of all positive transitions, etc.).

Example:

\begin{tabular}{llll}
\hline $\begin{array}{l}\text { Average positive transitions per } \\
\text { participant }=395\end{array}$ & & Transition Probability \\
positive to neutral transitions $=103$ & $\rightarrow$ & $.261(103 / 395)$ \\
positive to positive transitions $=156$ & $\rightarrow$ & $.395(156 / 395)$ \\
positive to negative transitions $=136$ & $\rightarrow$ & $.344(136 / 395)$ \\
\hline
\end{tabular}

\section{References}

Bousfield, W. A. (1953). The occurrence of clustering in the recall of randomly arranged associates. The Journal of General Psychology, 49, 229-240.

Bower, G. H. (1981). Mood and memory. American Psychologist, 36, 129-148.

Bradley, M. M., Greenwald, M. K., Petry, M. C., \& Lang, P. J. (1992). Remembering pictures: Pleasure and arousal in memory. Journal of Experimental Psychology, 18, 379-390.

Bradley, M. M., \& Lang, P. J. (1999). Affective norms for English words (ANEW): Stimuli, instruction manual and affective ratings (Tech. Rep. C-1). Gainesville: University of Florida, Center for Research in Psychophysiology.

Bradley, M. M., \& Lang, P. J. (2000). Affective reactions to acoustic stimuli. Psychophysiology, 37, 204-215.

Brosch, T., Pourtois, G., \& Sander, D. (2010). The perception and categorization of emotional stimuli: A review. Cognition \& Emotion, 24, 377-400.

Clark, J. M., \& Paivio, A. (2004). Extensions of the Paivio, Yuille, and Madigan (1968) norms. Behavior Research Methods, Instruments, \& Computers, 36, 371-383.
Doerksen, S., \& Shimamura, A. P. (2001). Source memory enhancement for emotional words. Emotion, 1, 5-11.

Easterbrook, J. A. (1959). The effect of emotion on cue utilization and the organization of behavior. Psychological Review, 66, 183-201.

Howard, M. W., \& Kahana, M. J. (1999). Contextual variability and serial position effects in free recall. Journal of Experimental Psychology: Human Learning and Memory, 25, 923-941.

Howard, M. W., \& Kahana, M. J. (2002). When does semantic similarity help episodic retrieval? Journal of Memory and Language, 46, 85-98.

Hunt, R. R., \& McDaniel, M. A. (1993). The enigma of organization and distinctiveness. Journal of Memory and Language, 32, 421445.

Kahana, M. J. (1996). Associative retrieval processes in free recall. Memory \& Cognition, 24, 103-109.

Kensinger, E. A. (2009). Remembering the details: Effects of emotion. Emotion Review, 1, 99-113.

Kensinger, E. A., \& Corkin, S. (2004). Two routes to emotional memory: Distinct neural processes for valence and arousal. Proceedings of the National Academy of Sciences, 101, 3310-3315.

Lang, P. J., Bradley, M. M., \& Cuthbert, B. N. (1990). Emotion, attention, and the startle reflex. Psychological Review, 97, 377395.

Levine, L. J., \& Edelstein, R. S. (2009). Emotion and memory narrowing: A review and goal relevance approach. Cognition \& Emotion, 23, 833-875.

Maddock, R. J., \& Frein, S. T. (2009). Reduced memory for spatial and temporal context of unpleasant words. Cognition \& Emotion, 23, 96-117.

Maltzman, I., Kantor, W., \& Langdon, B. (1966). Immediate and delayed retention, arousal, and the orienting and defensive reflexes. Psychonomic Sciences, 6, 445-446.

Mather, M. (2007). Emotional arousal and memory binding: An object-based framework. Perspectives on Psychological Science, 2, 33-52.

Niedenthal, P. M., Halberstadt, J. B., \& Innes-Ker, A. H. (1999). Emotional response categorization. Psychological Review, 106, $337-361$

Polyn, S. M., Norman, K. A., \& Kahana, M. J. (2009a). A context maintenance and retrieval model of organizational processes in free recall. Psychological Review, 116, 129-156.

Polyn, S. M., Norman, K. A., \& Kahana, M. J. (2009b). Task context and organization in free recall. Neuropsychologia, 47, 2158-2163.

Raaijmakers, J. G. W., \& Shiffrin, R. M. (1980). SAM: A theory of probabilistic search of associative memory. In G. H. Bower (Ed.), The psychology of learning and motivation (Vol. 14, pp. 207262). New York: Academic Press.

Roenker, D. L., Thompson, C. P., \& Brown, S. C. (1971). Comparison of measures for the estimation of clustering in free recall. Psychological Bulletin, 76, 45-48.

Rohrer, D. (1996). On the relative and absolute strength of a memory trace. Memory \& Cognition, 24, 188-201.

Schmidt, K., Patnaik, P., \& Kensinger, E. A. (2011). Emotion's influence on memory for spatial and temporal context. Cognition \& Emotion, 25, 229-243.

Schmidt, S. R., \& Saari, B. (2007). The emotional memory effect: Differential processing or item distinctiveness? Memory \& Cognition, 35, 1905-1916.

Sederberg, P. B., Miller, J. F., Howard, M. W., \& Kahana, M. J. (2010). The temporal contiguity effect predicts episodic memory performance. Memory \& Cognition, 38, 689-699.

Shiffrin, R. M. (1970). Memory search. In D. A. Norman (Ed.), Models of human memory (pp. 375-447). New York: Academic Press.

Sison, J. G., \& Mather, M. (2007). Does remembering emotional items impair recall of same-emotion items? Psychological Bulletin \& Review, 14, 282-287. 
Talmi, D., Luk, B. T. C., McGarry, L. M., \& Moscovitch, M. (2007). The contribution of relatedness and distinctiveness to emotionallyenhanced memory. Journal of Memory and Language, 56, 555-574.

Talmi, D., \& Moscovitch, M. (2004). Can semantic relatedness explain the enhancement of memory for emotional words? Memory \& Cognition, 32, 742-751.

Talmi, D., Schimmack, U., Paterson, T., \& Moscovitch, M. (2007). The role of attention and relatedness in emotionally enhanced memory. Emotion, 7, 89-102.

Unsworth, N. (2008). Exploring the retrieval dynamics of delayed and final free recall: Further evidence for temporal-contextual search. Journal of Memory and Language, 59, 223-236.
Unsworth, N. (2009). Variation in working memory capacity, fluid intelligence, and episodic recall: A latent variable examination of differences in the dynamics of free-recall. Memory \& Cognition, 37, 837-849.

Wixted, J. T., Ghadisha, H., \& Vera, R. (1997). Recall latency following pure- and mixed-strength lists: A direct test of the relative strength model of free recall. Journal of Experimental Psychology. Learning, Memory, and Cognition, 23, $523-538$.

Yiend, J. (2010). The effects of emotion on attention: A review of attentional processing of emotional information. Cognition \& Emotion, 24, 3-47. 